\title{
THE ASYMPTOTICS OF A SOLUTION OF THE MULTIDIMENSIONAL HEAT EQUATION WITH UNBOUNDED INITIAL DATA ${ }^{1}$
}

\author{
Sergey V. Zakharov \\ Krasovskii Institute of Mathematics and Mechanics, \\ Ural Branch of the Russian Academy of Sciences, \\ 16 S. Kovalevskaya str., Ekaterinburg, 620108 Russia \\ svz@imm.uran.ru
}

\begin{abstract}
For the multidimensional heat equation, the long-time asymptotic approximation of the solution of the Cauchy problem is obtained in the case when the initial function grows at infinity and contains logarithms in its asymptotics. In addition to natural applications to processes of heat conduction and diffusion, the investigation of the asymptotic behavior of the solution of the problem under consideration is of interest for the asymptotic analysis of equations of parabolic type. The auxiliary parameter method plays a decisive role in the investigation.
\end{abstract}

Key words: Multidimensional heat equation, Cauchy problem, Asymptotics, Auxiliary parameter method.

\section{Introduction}

In 1822, J. Fourier published his most fundamental work [4], where the heat conduction equation was presented and analyzed. This event provided a strong impetus for later researches in the fields of partial differential equations and trigonometric series. The famous equation has been further successfully used for effective descriptions of molecular diffusion, stochastic motion, the capillary conduction of liquids in porous media, and even for the analysis of social economic data. Already Fourier himself pointed out the universality of this mathematical model sine qua non in his eminent book as follows: "Il est facile de juger combien ces recherches intéressent les sciences physiques et l'économie civile, et quelle peut être leur influence sur les progrès des arts qui exigent l'emploi et la distribution du feu." ${ }^{2}$ Fourier's preliminary theoretical studying of heat phenomena and some vivid particulars of his elaborations in early 1800s are expressively reflected in the prefatory part of [4]. The historical survey [10] supplied with appropriate general and specialized references depicts many significant details of the subsequent life of the heat equation during the XIX and XX centuries.

Since the literature about the heat equation, in particular, and parabolic equations, in general, is immense, it is impossible in this introduction to give a complete picture of available results, and the bibliography below is of course by no means exhaustive. Here, we mention that existence and uniqueness theorems were obtained for a wide class of parabolic equations and systems [6, 15, 18, 19]; some results for unbounded solutions were presented in [11, 13]. As for the long-time behavior of solutions, we see that their stabilization, certain estimates, and the leading terms of asymptotics

\footnotetext{
${ }^{1}$ Dedicated to the 200th anniversary of Charles Hermite and "Théorie analytique de la chaleur" by Joseph Fourier.

2 "It is easy to judge how much these researches are interesting for the physical sciences and the civil economy and what may be their influence on the progress of the arts which require the employment and the distribution of fire."
} 
were mainly considered $[2,8,12,17]$. Complete asymptotic expansions of solutions into infinite series in inverse integer powers of the time variable were earlier obtained by Friedman in [5] and [6, Ch. 6] for bounded space-domains.

In the present paper, the long-time asymptotics of the solution of the Cauchy problem for the multidimensional heat equation

$$
\begin{gathered}
\frac{\partial u}{\partial t}=\frac{\partial^{2} u}{\partial x_{1}^{2}}+\ldots+\frac{\partial^{2} u}{\partial x_{m}^{2}}, \quad t>0, \quad m \geqslant 2, \\
u\left(x_{1}, \ldots, x_{m}, 0\right)=\Lambda\left(x_{1}, \ldots, x_{m}\right), \quad\left(x_{1}, \ldots, x_{m}\right) \in \mathbb{R}^{m},
\end{gathered}
$$

is obtained for a locally Lebesgue integrable initial function $\Lambda: \mathbb{R}^{m} \rightarrow \mathbb{R}$ of polynomial growth. As is well known [18], in the class of smooth functions of moderate growth for $t>0$, there exists a unique solution of problem (1.1)-(1.2) and it can be written in the form of the Poisson integral ${ }^{3}$

$$
u(x, t)=\frac{1}{(4 \pi t)^{m / 2}} \int_{\mathbb{R}^{m}} \Lambda(s) \exp \left(-\frac{|s-x|^{2}}{4 t}\right) d s,
$$

where $x=\left(x_{1}, \ldots, x_{m}\right) \in \mathbb{R}^{m}, s=\left(s_{1}, \ldots, s_{m}\right) \in \mathbb{R}^{m}$, and $d s=d s_{1} \ldots d s_{m}$.

It should be noted that the investigation of the asymptotic behavior of the function $u(x, t)$, in addition to possible natural applications to the modeling of physical processes of heat conduction and diffusion, may be of interest for the asymptotic analysis of solutions of nonlinear parabolic equations by the matching method $[9,21]$ as well as for the theory of invariants [7] and some issues of matrix geometry [14].

Below, a complete asymptotic expansion of the solution $u(x, t)$ of problem (1.1)-(1.2) is found as $|x|+t \rightarrow+\infty$ under the following suppositions:

$$
\begin{gathered}
\Lambda\left(x_{1}, \ldots, x_{m}\right)=0, \quad x_{1}<0, \\
\Lambda\left(x_{1}, \ldots, x_{m}\right)=x_{1}^{p} \sum_{n=0}^{\infty} x_{1}^{-n} \sum_{j=0}^{n} \Lambda_{n, j}\left(x^{\prime}\right) \ln ^{j} x_{1}, \quad x_{1} \rightarrow+\infty,
\end{gathered}
$$

where $p$ is a positive integer and $\Lambda_{n, j}\left(x^{\prime}\right)$ are Lebesgue integrable functions of $x^{\prime}=\left(x_{2}, \ldots, x_{m}\right)$; for simplicity, we also suppose that

$$
\begin{gathered}
\operatorname{supp} \Lambda \subset\left\{\left(x_{1}, \ldots, x_{m}\right): x_{1}>0,\left|x_{2}\right|+\ldots+\left|x_{m}\right|<x_{1}^{\nu}\right\}, \quad \nu>0, \\
\operatorname{supp} \Lambda_{n, j} \subset\left\{\left(x_{2}, \ldots, x_{m}\right):\left|x_{2}\right|+\ldots+\left|x_{m}\right|<r_{n}\right\}, \quad r_{n}>0 .
\end{gathered}
$$

Although $\Lambda$ is a function of several variables, the asymptotic series (1.5) must be understood here in the usual sense of Poincaré $[16, \S 1]$ due to the second condition $(1.6)$, that is

$$
\Lambda\left(x_{1}, \ldots, x_{m}\right)=\sum_{n=0}^{N-1} x_{1}^{p-n} \sum_{j=0}^{n} \Lambda_{n, j}\left(x^{\prime}\right) \ln ^{j} x_{1}+O\left(x_{1}^{p-N} \ln ^{N} x_{1}\right), \quad x_{1} \rightarrow+\infty,
$$

for any integer $N \geqslant 1$. It should be also said that the appearance of asymptotic series of form (1.5) is typical for the matching method [9].

The main difficulty of the calculation of the asymptotic expansion of integral (1.3) is exactly due to condition (1.5) and the "smearing" of the integrand exponent as $t \rightarrow+\infty$; if we formally put $t=+\infty$, then we generally get the divergence of the integral. Thus, the asymptotic limit under consideration is diametrically opposite to the well-known case of the integrals of Laplace's type with the sharpening exponent and a suitable computational technique suggested by Danilin in [1] is therefore complementary to the standard Laplace method. This technique is called the auxiliary parameter method.

\footnotetext{
${ }^{3}$ In essence, this solution was given by Fourier [4, Ch. IX, §392].
} 


\section{Applying the auxiliary parameter method}

To obtain the asymptotic behavior of integral (1.3) as the space-time variables $(x, t)$ independently tend to infinity, we apply a scheme similar to that used in [20] for the solution of the heat equation in $\mathbb{R}_{x}^{1} \times \mathbb{R}_{t}^{+}$. First of all, taking into account condition (1.4), we represent function (1.3) in the form of the sum

$$
u(x, t)=U_{0}(x, t)+U_{1}(x, t),
$$

where

$$
\begin{gathered}
U_{0}(x, t)=\int_{0}^{\sigma(x, t)} \int_{\mathbb{R}^{m-1}} \ldots d s^{\prime} d s_{1}, \quad U_{1}(x, t)=\int_{\sigma(x, t)}^{+\infty} \int_{\mathbb{R}^{m-1}} \ldots d s^{\prime} d s_{1}, \\
\sigma(x, t)=\left(|x|^{2}+t\right)^{\beta / 2}, \quad 0<\beta<1,
\end{gathered}
$$

the dots denote the integrand in formula (1.3) together with the factor $(4 \pi t)^{-m / 2}$, the number $\beta$ is an arbitrary parameter, and $d s^{\prime}=d s_{2} \ldots d s_{m}$. Under conditions (1.4) and (1.5), the asymptotics of the integrals $U_{0}(x, t)$ and $U_{1}(x, t)$ can be computed by using the expansions of the kernel exponent and the initial function $\Lambda$, respectively.

\subsection{Asymptotics of $U_{1}(x, t)$}

In the integral $U_{1}(x, t)$, we make the change $s_{1}=2 z \sqrt{t}$ and put

$$
\mu(x, t)=\frac{\sigma(x, t)}{2 \sqrt{t}}, \quad \eta_{1}=\frac{x_{1}}{2 \sqrt{t}} .
$$

Next, using condition (1.5), for any integer $N \geqslant p+1$, we obtain (hereinafter we often omit the arguments of $\sigma$ and $\mu$ )

$$
\begin{aligned}
U_{1}(x, t)=\frac{1}{\pi^{m / 2}(4 t)^{(m-1) / 2}} \int_{\mu}^{+\infty} \exp \left(-\left(\eta_{1}-z\right)^{2}\right) \int_{\mathbb{R}^{m-1}} \Lambda\left(2 z \sqrt{t}, s^{\prime}\right) \exp \left(-\frac{\left|s^{\prime}-x^{\prime}\right|^{2}}{4 t}\right) d s^{\prime} d z \\
=\frac{t^{p / 2}}{\sqrt{\pi}} \sum_{n=0}^{N-1} 2^{p-n} t^{-n / 2} \sum_{j=0}^{n} \int_{\mu}^{+\infty} z^{p-n} \ln ^{j}(2 z \sqrt{t}) \exp \left(-\left(z-\eta_{1}\right)^{2}\right) d z \\
\quad \times \frac{1}{(4 \pi t)^{(m-1) / 2}} \int_{\mathbb{R}^{m-1}} \Lambda_{n, j}\left(s^{\prime}\right) \exp \left(-\frac{\left|s^{\prime}-x^{\prime}\right|^{2}}{4 t}\right) d s^{\prime}+R(x, t),
\end{aligned}
$$

where

$$
|R(x, t)| \leqslant \frac{M_{N}}{\sqrt{t}} \int_{\sigma}^{+\infty} s_{1}^{p-N} \ln ^{N} s_{1} \exp \left(-\frac{\left(s_{1}-x_{1}\right)^{2}}{4 t}\right) d s_{1}, \quad M_{N}>0,
$$

by formula (1.7). Then, for $N \geqslant p+1$, we have

$$
\begin{aligned}
U_{1}(x, t)= & \frac{t^{p / 2}}{\sqrt{\pi}} \sum_{n=0}^{N-1} 2^{p-n} t^{-n / 2} \sum_{j=0}^{n} \sum_{l=0}^{j} \frac{j ! \ln ^{l} t}{2^{l} l !(j-l) !} \int_{\mu}^{+\infty} z^{p-n} \ln ^{j-l}(2 z) \exp \left(-\left(z-\eta_{1}\right)^{2}\right) d z \\
& \times \frac{1}{(4 \pi t)^{(m-1) / 2}} \int_{\mathbb{R}^{m-1}} \Lambda_{n, j}\left(s^{\prime}\right) \exp \left(-\frac{\left|s^{\prime}-x^{\prime}\right|^{2}}{4 t}\right) d s^{\prime}+O\left(\sigma^{p-N} \ln ^{N} \sigma\right)
\end{aligned}
$$


as $\sigma=\sigma(x, t) \rightarrow+\infty$. Changing the order of summation, we find

$$
\begin{aligned}
& U_{1}(x, t)=t^{p / 2} \sum_{n=0}^{N-1} t^{-n / 2} \sum_{l=0}^{n} \ln ^{l} t \sum_{j=l}^{n} \frac{j ! 2^{p-n-l}}{\sqrt{\pi} l !(j-l) !} \int_{\mu}^{+\infty} z^{p-n} \ln ^{j-l}(2 z) \exp \left(-\left(z-\eta_{1}\right)^{2}\right) d z \\
& \times \frac{1}{(4 \pi t)^{(m-1) / 2}} \int_{\mathbb{R}^{m-1}} \Lambda_{n, j}\left(s^{\prime}\right) \exp \left(-\frac{\left|s^{\prime}-x^{\prime}\right|^{2}}{4 t}\right) d s^{\prime}+O\left(\sigma^{p-N} \ln ^{N} \sigma\right), \quad \sigma \rightarrow+\infty .
\end{aligned}
$$

To handle the integral with respect to $z$, it is convenient to consider first the following set of independent variables:

$$
T_{\alpha}=\left\{(x, t): x \in \mathbb{R}^{m}, t \geqslant|x|^{\alpha}>1,1+\beta<\alpha<2\right\} .
$$

The obvious inequalities

$$
\sigma(x, t) \leqslant\left(t^{2 / \alpha}+t\right)^{\beta / 2}<2^{\beta / 2} t^{\beta / \alpha}
$$

for $(x, t) \in T_{\alpha}$ imply that

$$
t>2^{-\alpha / 2}[\sigma(x, t)]^{\alpha / \beta} \quad \text { for }(x, t) \in T_{\alpha} ;
$$

therefore, on account of the first definition (2.3), we obtain

$$
0<\mu(x, t)<2^{\alpha / 4-1}[\sigma(x, t)]^{-\gamma} \quad \text { for } \quad(x, t) \in T_{\alpha}, \quad \text { where } \quad \gamma=\frac{\alpha}{2 \beta}-1>0 .
$$

For $0 \leqslant n \leqslant p$, we have

$$
\begin{aligned}
\int_{\mu}^{+\infty} z^{p-n} \ln ^{j-l}(2 z) e^{-\left(z-\eta_{1}\right)^{2}} d z & =\int_{0}^{+\infty} z^{p-n} \ln ^{j-l}(2 z) e^{-\left(z-\eta_{1}\right)^{2}} d z-\int_{0}^{\mu} z^{p-n} \ln ^{j-l}(2 z) e^{-\left(z-\eta_{1}\right)^{2}} d z \\
& =\int_{-\eta_{1}}^{+\infty}\left(\eta_{1}+s\right)^{p-n} \ln ^{j-l}\left(2\left(\eta_{1}+s\right)\right) e^{-s^{2}} d s-\int_{0}^{\mu} z^{p-n} \ln ^{j-l}(2 z) e^{-\left(z-\eta_{1}\right)^{2}} d z .
\end{aligned}
$$

Since by (2.7) $\mu \rightarrow+0$ as $\sigma \rightarrow+\infty$ for $(x, t) \in T_{\alpha}$, it follows that

$$
\begin{gathered}
\int_{\mu}^{+\infty} z^{p-n} \ln ^{j-l}(2 z) e^{-\left(z-\eta_{1}\right)^{2}} d z=\sum_{q=0}^{p-n} \frac{(p-n) ! \eta_{1}^{p-n-q}}{q !(p-n-q) !} \int_{-\eta_{1}}^{+\infty} s^{q} \ln ^{j-l}\left[2\left(\eta_{1}+s\right)\right] e^{-s^{2}} d s \\
+e^{-\eta_{1}^{2}} \sum_{s: r_{s}^{2}+l_{s}^{2} \neq 0} b_{s}^{\prime} \eta_{1}^{n_{s}} \mu^{r_{s}} \ln ^{l_{s}} \mu+O\left(\sigma^{-\gamma N}\right), \quad \sigma \rightarrow+\infty,
\end{gathered}
$$

where the finite sum over $s$ with $b_{s}^{\prime}$ being some constants and $n_{s}, r_{s}, l_{s}$ being some nonnegative integers depends naturally on $N$. For $n>p$, we have

$$
\begin{gathered}
\int_{\mu}^{+\infty} z^{p-n} \ln ^{j-l}(2 z) e^{-\left(z-\eta_{1}\right)^{2}} d z=\int_{1}^{+\infty} z^{p-n} \ln ^{j-l}(2 z) e^{-\left(z-\eta_{1}\right)^{2}} d z \\
+\int_{\mu}^{1} \ln ^{j-l}(2 z) \Psi_{n-p}\left(z, \eta_{1}\right) d z+e^{-\eta_{1}^{2}} \sum_{r=0}^{n-p-1} P_{r}\left(\eta_{1}\right) \int_{\mu}^{1} \ln ^{j-l}(2 z) z^{r+p-n} d z,
\end{gathered}
$$


where $P_{r}\left(\eta_{1}\right)$ are some polynomials of degree $r$,

$$
\Psi_{n-p}\left(z, \eta_{1}\right)=z^{p-n}\left[e^{-\left(z-\eta_{1}\right)^{2}}-e^{-\eta_{1}^{2}} \sum_{r=0}^{n-p-1} H_{r}\left(\eta_{1}\right) \frac{z^{r}}{r !}\right],
$$

and the sum in the square brackets is a partial sum of the Maclaurin series for the function $\exp \left(2 z \eta_{1}-z^{2}\right)$ in variable $z$ with $H_{r}\left(\eta_{1}\right)$ being the Hermite polynomials of degree $r$. This implies the equality

$$
\begin{gathered}
\int_{\mu}^{+\infty} z^{p-n} \ln ^{j-l}(2 z) e^{-\left(z-\eta_{1}\right)^{2}} d z \\
=\int_{1}^{+\infty} z^{p-n} \ln ^{j-l}(2 z) e^{-\left(z-\eta_{1}\right)^{2}} d z+e^{-\eta_{1}^{2}} \sum_{s: r_{s}^{2}+l_{s}^{2} \neq 0} b_{s}^{\prime \prime} \eta_{1}^{n_{s}} \mu^{r_{s}} \ln ^{l_{s}} \mu \\
\quad+\int_{0}^{1} \ln ^{j-l}(2 z) \Psi_{n-p}\left(z, \eta_{1}\right) d z-\int_{0}^{\mu} \ln ^{j-l}(2 z) \Psi_{n-p}\left(z, \eta_{1}\right) d z
\end{gathered}
$$

with $b_{s}^{\prime \prime}$ being some constants and $n_{s}, r_{s}, l_{s}$ being some nonnegative integers. From formula (2.9) we easily conclude that the function $\Psi_{n-p}\left(z, \eta_{1}\right)$ has no singularities as $z \rightarrow 0$; therefore, the last two integrals in (2.10) converge and relation (2.10) itself thus becomes

$$
\int_{\mu}^{+\infty} z^{p-n} \ln ^{j-l}(2 z) e^{-\left(z-\eta_{1}\right)^{2}} d z=J_{p, n, j, l}\left(\eta_{1}\right)+e^{-\eta_{1}^{2}} \sum_{s: r_{s}^{2}+l_{s}^{2} \neq 0} b_{s}^{\prime \prime \prime} \eta_{1}^{n_{s}} \mu^{r_{s}} \ln ^{l_{s}} \mu+O\left(\sigma^{-\gamma N}\right)
$$

as $\sigma \rightarrow+\infty$, where

$$
J_{p, n, j, l}\left(\eta_{1}\right)=\int_{1}^{+\infty} z^{p-n} \ln ^{j-l}(2 z) e^{-\left(z-\eta_{1}\right)^{2}} d z+\int_{0}^{1} \ln ^{j-l}(2 z) \Psi_{n-p}\left(z, \eta_{1}\right) d z,
$$

$b_{s}^{\prime \prime \prime}$ are some constants, $n_{s}, r_{s}, l_{s}$ are some nonnegative integers, and $\gamma$ is defined in (2.7).

Using the second condition (1.6) and Maclaurin's expansion for the exponent in the integrand of $(2.4)$ in $s^{\prime} t^{-1 / 2}$, for any natural $N^{*} \geqslant 1$, we obtain

$$
\begin{gathered}
\frac{1}{(4 \pi t)^{(m-1) / 2}} \int_{\mathbb{R}^{m-1}} \Lambda_{n, j}\left(s^{\prime}\right) \exp \left(-\frac{\left|s^{\prime}-x^{\prime}\right|^{2}}{4 t}\right) d s^{\prime} \\
=t^{(1-m) / 2} \exp \left(-\left|\eta^{\prime}\right|^{2}\right)\left[\sum_{l=0}^{N^{*}-1} t^{-l / 2} Q_{l}^{(n, j)}\left(\eta^{\prime}\right)+O\left(t^{-N^{*} / 2}\left|\eta^{\prime}\right|^{N^{*}}\right)\right],
\end{gathered}
$$

where $Q_{l}^{(n, j)}\left(\eta^{\prime}\right)$ are some $l$ th degree polynomials in $\eta^{\prime}=2^{-1} t^{-1 / 2} x^{\prime}$ whose coefficients depend on $n$ and $j$. Substituting expressions (2.8), (2.11), and (2.13) into formula (2.4) and taking into account that $\sigma^{-(\gamma+\alpha / 2 \beta) N}=O\left(\sigma^{-N}\right)$, since $\gamma+\alpha / 2 \beta=\alpha / \beta-1>1$, we find that

$$
U_{1}(x, t)=t^{(p-m+1) / 2} \sum_{n=0}^{N-1} t^{-n / 2} \sum_{l=0}^{n} \widetilde{S}_{n, l}(\eta) \ln ^{l} t+V_{1, N}(\mu, \eta, t)+O\left(\sigma^{p-N} \ln ^{N} \sigma\right)
$$


as $\sigma \rightarrow+\infty$, where, according to formulas (2.9) and (2.12), the coefficients $\widetilde{S}_{n, l}(\eta)$ are some smooth functions of polynomial growth for $0 \leqslant n \leqslant p$ and of superexponential decreasing for $n>p$,

$$
V_{1, N}(\mu, \eta, t)=\exp \left(-|\eta|^{2}\right) \sum_{s: r_{s}^{2}+l_{s}^{2} \neq 0} a_{s}^{\prime} t^{k_{s}} \eta^{n_{s}} \mu^{r_{s}} \ln ^{l_{s}} \mu
$$

is a finite sum with $\eta^{n_{s}}=\eta_{1}^{n_{1, s}} \ldots \eta_{m}^{n_{m, s}}, a_{s}^{\prime}$ being some real constants, $k_{s}$ being half-integer numbers, and $n_{j, s}, r_{s}, l_{s}$ being some nonnegative integers. Because of the factor $\exp \left(-|\eta|^{2}\right)$, the estimate of the remainder in formula (2.14) remains true for the values of the independent variables from the set

$$
X_{\alpha}=\left\{(x, t):|x|>1,0<t<|x|^{\alpha}\right\},
$$

since for $(x, t) \in X_{\alpha}$ there hold the following inequalities:

$$
\mu^{2}=\frac{\left(|x|^{2}+t\right)^{\beta}}{4 t}<2|\eta|^{2}|x|^{-2(1-\beta)}, \quad|\eta|^{2}>\frac{1}{4}|x|^{2-\alpha}>\frac{1}{8} \sigma^{(2-\alpha) / \beta} .
$$

\subsection{Asymptotics of $U_{0}(x, t)$}

Now, let us pass to the evaluation of the integral

$$
U_{0}(x, t)=\frac{1}{(4 \pi t)^{m / 2}} \int_{0}^{\sigma(x, t)} d s_{1} \int_{\mathbb{R}^{m-1}} d s^{\prime} \Lambda\left(s_{1}, s^{\prime}\right) \exp \left(-\frac{|s-x|^{2}}{4 t}\right) d s .
$$

From the obvious inequality $|x|^{2} \leqslant[\sigma(x, t)]^{2 / \beta}$ and inequality (2.6) we conclude that

$$
\frac{|s|^{2}}{t}=O\left(\sigma^{-2 \delta}\right), \quad \frac{x_{k} s_{k}}{t}=\frac{2 \eta_{k} s_{k}}{\sqrt{t}}=O\left(\sigma^{-\delta}\right), \quad \delta=\frac{\alpha-1}{\beta}-1>0,
$$

for $|s| \leqslant \sigma$ and $(x, t) \in T_{\alpha}$, where $1 \leqslant k \leqslant m$. Then, using conditions (1.6), (1.7) and estimates (2.18), we represent the integral $U_{0}(x, t)$ in the following form:

$U_{0}(x, t)=\frac{\exp \left(-|\eta|^{2}\right)}{(4 \pi t)^{m / 2}}\left[\int_{0}^{\sigma} \int_{\mathbb{R}^{m-1}} \Lambda\left(s_{1}, s^{\prime}\right) \sum_{q=0}^{N-1} \frac{1}{q !}\left(\frac{\eta_{1} s_{1}+\ldots+\eta_{m} s_{m}}{\sqrt{t}}-\frac{|s|^{2}}{4 t}\right)^{q} d s^{\prime} d s_{1}+O\left(\sigma^{p+1-\delta N}\right)\right]$

as $\sigma \rightarrow+\infty$ with any $N \geqslant 1$. Because of the factor $\exp \left(-|\eta|^{2}\right)$, the estimate of the remainder holds also true on the set $X_{\alpha}$ defined by (2.16). Expanding the parenthesis in the above formula for $U_{0}(x, t)$ and changing the order of summation, we obtain

$$
\begin{gathered}
U_{0}(x, t)=\frac{\exp \left(-|\eta|^{2}\right)}{t^{m / 2}} \sum_{n=0}^{N-1} t^{-n / 2} \sum_{\substack{0 \leqslant k_{1}+\ldots+k_{m} \leqslant n \\
0 \leqslant l_{1}+l_{2,2}+\ldots+l_{2, m} \leqslant n}} a_{k, l} \eta^{k} \int_{0}^{\sigma} \int_{\mathbb{R}^{m-1}} s_{1}^{l_{1}}\left(s^{\prime}\right)^{l_{2}} \Lambda\left(s_{1}, s^{\prime}\right) d s^{\prime} d s_{1} \\
+O\left(\frac{\exp \left(-|\eta|^{2}\right)}{t^{m / 2}} \sigma^{p+1-\delta N}\right)
\end{gathered}
$$

as $\sigma \rightarrow+\infty$, where $a_{k, l}=a_{k_{1}, \ldots, k_{m}, l_{1}, l_{2,2}, \ldots, l_{2, m}}$ are some constants, $\eta^{k}=\eta_{1}^{k_{1}} \ldots \eta_{m}^{k_{m}}$, and $\left(s^{\prime}\right)^{l_{2}}=s_{2}^{l_{2,2}} \ldots s_{m}^{l_{2, m}}$. Keeping in mind the asymptotic condition (1.7), we transform the multiple integral appeared above as follows:

$$
\int_{0}^{\sigma} \int_{\mathbb{R}^{m-1}} s_{1}^{l_{1}}\left(s^{\prime}\right)^{l_{2}} \Lambda\left(s_{1}, s^{\prime}\right) d s^{\prime} d s_{1}=\int_{0}^{1} \int_{\mathbb{R}^{m-1}} s_{1}^{l_{1}}\left(s^{\prime}\right)^{l_{2}} \Lambda\left(s_{1}, s^{\prime}\right) d s^{\prime} d s_{1}
$$




$$
\begin{gathered}
+\int_{1} \int_{\mathbb{R}^{m-1}} s_{1}^{l_{1}}\left(s^{\prime}\right)^{l_{2}}\left[\Lambda\left(s_{1}, s^{\prime}\right)-\sum_{q=0}^{p+l_{1}+1} s_{1}^{p-q} \sum_{j=0}^{q} \Lambda_{q, j}\left(s^{\prime}\right) \ln ^{j} s_{1}\right] d s^{\prime} d s_{1} \\
+\int_{1}^{\sigma} \int_{\mathbb{R}^{m-1}}\left[\left(s^{\prime}\right)^{l_{2}} \sum_{q=0}^{p+l_{1}+1} s_{1}^{p-q+l_{1}} \sum_{j=0}^{q} \Lambda_{q, j}\left(s^{\prime}\right) \ln ^{j} s_{1}\right] d s^{\prime} d s_{1} \\
=\sum_{j=0}^{p+l_{1}+1} c_{l_{1}, l_{2}, j} \ln ^{j+1} \sigma+\sum_{i, j: i \neq 0} c_{l_{1}, l_{2}, i, j} \sigma^{i} \ln ^{j} \sigma+O\left(\sigma^{-N^{*}} \ln ^{N^{*}} \sigma\right), \quad \sigma \rightarrow+\infty
\end{gathered}
$$

with $c_{l_{1}, l_{2}, j}$ and $c_{l_{1}, l_{2}, i, j}^{*}$ being some constants, where the finite sum over $i, j$ depends naturally on a sufficiently large $N^{*}$; here we used the elementary relation

$$
\int_{1}^{\sigma} s_{1}^{k} \ln ^{j} s_{1} d s_{1}=\sigma^{k+1} \sum_{l=0}^{j-1} \frac{(-1)^{l} j ! \ln ^{j-l} \sigma}{(k+1)^{l+1}(j-l) !}+\left(\sigma^{k+1}-1\right) \frac{(-1)^{j} j !}{(k+1)^{j+1}} \quad(k \geqslant 0, \quad j \geqslant 1) .
$$

From formulas (2.3), inequality (2.6), the uniform estimate

$$
t^{-m / 2} \eta^{n} \exp \left(-|\eta|^{2}\right)=O\left(\sigma^{-\alpha m / 2 \beta}\right)
$$

and the previous asymptotic expression for $U_{0}(x, t)$, it follows that

$$
U_{0}(x, t)=\frac{\exp \left(-|\eta|^{2}\right)}{t^{m / 2}} \sum_{n=0}^{N-1} t^{-n / 2} \sum_{j=0}^{p+n+2} \Pi_{n, j}(\eta) \ln ^{j} t+V_{0, N}(\mu, \eta, t)+O\left(\sigma^{p+1-\delta N}\right)
$$

as $\sigma \rightarrow+\infty$, where $\delta$ is defined in (2.18), $\Pi_{n, j}(\eta)$ are some polynomials of degree $n$, and the finite sum

$$
V_{0, N}(\mu, \eta, t)=\exp \left(-|\eta|^{2}\right) \sum_{s: r_{s}^{2}+l_{s}^{2} \neq 0} a_{s}^{\prime \prime} t^{k_{s}} \eta^{n_{s}} \mu^{r_{s}} \ln ^{l_{s}} \mu
$$

with $a_{s}^{\prime \prime}$ being some constants, is obtained similarly to expression (2.15).

\subsection{Evaluation of the "virtual terms"}

In the sequel, it is convenient to suppose that $1+\beta<\alpha<1+2 \beta$, whence we find the inequalities $0<\delta=(\alpha-1) / \beta-1<1$ and the asymptotic estimate $\sigma^{p-N} \ln ^{N} \sigma=O\left(\sigma^{p+1-\delta N}\right)$ as $\sigma \rightarrow+\infty$. Then substituting expansions (2.14) and (2.19) into formula (2.1), we summarize the results of the previous two subsections as follows.

Lemma 1. For the solution of the Cauchy problem (1.1)-(1.2), the asymptotic formula

$$
\begin{gathered}
u(x, t)=t^{-m / 2} \sum_{n=0}^{N-1} t^{-n / 2}\left[\sum_{l=0}^{n} t^{(p+1) / 2} \widetilde{S}_{n, l}(\eta) \ln ^{l} t+\sum_{j=0}^{p+n+2} \Pi_{n, j}(\eta) \exp \left(-|\eta|^{2}\right) \ln ^{j} t\right] \\
+V_{0, N}(\mu, \eta, t)+V_{1, N}(\mu, \eta, t)+O\left(\sigma^{p+1-\delta N}\right)
\end{gathered}
$$

holds true as $\sigma \rightarrow+\infty$, where $N \geqslant p+1, \widetilde{S}_{n, j}(\eta)$ are smooth functions of polynomial growth, $\Pi_{n, j}(\eta)$ are $n$th degree polynomials, and the functions $V_{0, N}(\mu, \eta, t)$ and $V_{1, N}(\mu, \eta, t)$ are defined by expressions (2.15) and (2.20). 
Now we must evaluate the "virtual terms" that depend on the value $\mu(x, t)=2^{-1} t^{-1 / 2}\left(|x|^{2}+t\right)^{\beta / 2}$ with the arbitrary parameter $\beta$.

From inequalities (2.17), we conclude that, for $(x, t) \in X_{\alpha}$, any integer numbers $n_{s, j}, r_{s}, l_{s}$, and half-integer number $k_{s}$, there exist $C>0$ and $q>0$ such that

$$
\left|t^{k_{s}} \eta_{j}^{n_{s, j}} \mu^{r_{s}} \ln ^{l_{s}} \mu\right| \exp \left(-|\eta|^{2}\right) \leqslant C \sigma^{q} \exp \left(-8^{-1} \sigma^{(2-\alpha) / \beta}\right) .
$$

Consequently, the expressions $V_{0, N}(\mu, \eta, t)$ and $V_{1, N}(\mu, \eta, t)$ in formulas (2.14) and (2.19) are exponentially small for $(x, t) \in X_{\alpha}$, since $\alpha<2$ by $(2.5)$.

For $(x, t) \in T_{\alpha}$, we introduce a small quantity $\varepsilon=\left(|x|^{2}+t\right)^{-1 / 4}$; whence, according to (2.2) and (2.3), we easily get the relations

$$
\sigma=\varepsilon^{-2 \beta}, \quad \mu=2^{-1} t^{-1 / 2} \varepsilon^{-2 \beta} .
$$

Then, by formulas (2.15), (2.20), and (2.22), we have

$$
V_{0, N}(\mu, \eta, t)+V_{1, N}(\mu, \eta, t)=\exp \left(-|\eta|^{2}\right) \sum_{s=1}^{L(N)} a_{s}^{\prime \prime \prime} t^{k_{s}} \ln ^{k_{s}^{\prime}} t \eta^{n_{s}} \varepsilon^{-2 \beta r_{s}} \ln ^{l_{s}} \varepsilon^{2 \beta}
$$

where $\varepsilon \rightarrow+0$ as $|x|^{2}+t \rightarrow+\infty, L(N) \in \mathbb{N}, a_{s}^{\prime \prime \prime}$ are some constants, $\eta^{n_{s}}=\eta_{1}^{n_{s, 1}} \ldots \eta_{m}^{n_{s, m}}, k_{s}$ are half-integer numbers, $k_{s}^{\prime}, n_{s, j}, r_{s}, l_{s}$, are nonnegative integers such that $r_{s}^{2}+l_{s}^{2} \neq 0$, and $\beta$ is an arbitrary parameter, without loss of generality, such that $0<\beta_{1} \leqslant \beta \leqslant \beta_{2}<1$, where $\beta_{1}<\beta_{2}$.

By virtue of the arbitrariness of the value $\beta$, from formulas (2.21) and (2.23) with $\beta=\beta_{1}$ and $\beta=\beta_{2}$ such that all numbers $2 \beta_{1} r_{1}, \ldots, 2 \beta_{1} r_{L(N)}, 2 \beta_{2} r_{1}, \ldots, 2 \beta_{2} r_{L(N)}$ are pairwise distinct, we obtain the following asymptotic relation with $r_{s}^{2}+l_{s}^{2} \neq 0$ :

$$
\exp \left(-|\eta|^{2}\right) \sum_{s=1}^{L(N)} a_{s}^{\prime \prime \prime} t^{k_{s}} \ln ^{k_{s}^{\prime}} t \eta^{n_{s}}\left(\varepsilon^{-2 \beta_{1} r_{s}} \ln ^{l_{s}} \varepsilon^{2 \beta_{1}}-\varepsilon^{-2 \beta_{2} r_{s}} \ln ^{l_{s}} \varepsilon^{2 \beta_{2}}\right)=O\left(\varepsilon^{2\left(\alpha-1-\beta_{1}\right) N-2 \beta_{1}(p+1)}\right)
$$

as $\varepsilon \rightarrow+0$. Consequently, taking into account the finiteness of the sum in the left-hand side, we have to conclude about every particular term in the left-hand side that either its order is not greater than the estimate in the right-hand side or the corresponding coefficient $a_{s}^{\prime \prime \prime}$ is equal to zero. Thus, we arrive at the following statement with $\beta=\beta_{1}$.

Lemma 2. For some $\beta \in(0,1)$ and $\alpha \in(1+\beta, 1+2 \beta)$, the asymptotic estimate

$$
V_{0, N}(\mu, \eta, t)+V_{1, N}(\mu, \eta, t)=O\left(\left(|x|^{2}+t\right)^{-(\alpha-1-\beta) N / 2+\beta(p+1) / 2}\right)
$$

holds true as $|x|^{2}+t \rightarrow+\infty$.

\section{Asymptotics of the solution}

Immediately from Lemmas 1 and 2, we obtain our main result.

Theorem 1. Let $u: \mathbb{R}^{m} \times \mathbb{R}^{+} \rightarrow \mathbb{R}$ be the solution of the Cauchy problem

$$
\begin{gathered}
\frac{\partial u}{\partial t}=\frac{\partial^{2} u}{\partial x_{1}^{2}}+\ldots+\frac{\partial^{2} u}{\partial x_{m}^{2}}, \quad t>0, \quad m \geqslant 2, \\
u\left(x_{1}, \ldots, x_{m}, 0\right)=\Lambda\left(x_{1}, \ldots, x_{m}\right), \quad\left(x_{1}, \ldots, x_{m}\right) \in \mathbb{R}^{m},
\end{gathered}
$$


with a locally Lebesgue integrable initial function $\Lambda: \mathbb{R}^{m} \rightarrow \mathbb{R}$. And let the following conditions be fulfilled:

$$
\begin{gathered}
\Lambda\left(x_{1}, \ldots, x_{m}\right)=0 \quad \text { for } \quad x_{1}<0, \\
\Lambda\left(x_{1}, \ldots, x_{m}\right)=x_{1}^{p} \sum_{n=0}^{\infty} x_{1}^{-n} \sum_{j=0}^{n} \Lambda_{n, j}\left(x_{2}, \ldots, x_{m}\right) \ln ^{j} x_{1} \quad \text { as } \quad x_{1} \rightarrow+\infty,
\end{gathered}
$$

where $p$ is a positive integer,

$$
\begin{gathered}
\operatorname{supp} \Lambda \subset\left\{\left(x_{1}, \ldots, x_{m}\right): x_{1}>0,\left|x_{2}\right|+\ldots+\left|x_{m}\right|<x_{1}^{\nu}\right\}, \quad \nu>0, \\
\operatorname{supp} \Lambda_{n, j} \subset\left\{\left(x_{2}, \ldots, x_{m}\right):\left|x_{2}\right|+\ldots+\left|x_{m}\right|<r_{n}\right\}, \quad r_{n}>0 .
\end{gathered}
$$

Then there holds the asymptotic formula

$u\left(x_{1}, \ldots, x_{m}, t\right)=t^{-m / 2} \sum_{n=0}^{\infty} t^{-n / 2} \sum_{j=0}^{p+n+2} \ln ^{j} t\left[t^{(p+1) / 2} S_{n, j}\left(\eta_{1}, \ldots, \eta_{m}\right)+\Pi_{n, j}\left(\eta_{1}, \ldots, \eta_{m}\right) \exp \left(-|\eta|^{2}\right)\right]$

as $\left|x_{1}\right|+\ldots+\left|x_{m}\right|+t \rightarrow+\infty$, where $S_{n, j}\left(\eta_{1}, \ldots, \eta_{m}\right)$ are smooth functions of polynomial growth and $\Pi_{n, j}\left(\eta_{1}, \ldots, \eta_{m}\right)$ are $n$th degree polynomials in the self-similar variables

$$
\eta_{1}=\frac{x_{1}}{2 \sqrt{t}}, \quad \ldots, \quad \eta_{m}=\frac{x_{m}}{2 \sqrt{t}}
$$

\section{Conclusion}

According to formulas (2.14), (2.19), and (2.24), the obtained expansion of the solution in Theorem 1 is understood in the sense of Erdélyi [3, Definition 2.4] with the gauge (asymptotic) sequence $\left\{\left(|x|^{2}+t\right)^{-\rho N}\right\}_{N=1}^{\infty}$, where $\rho>0$, that is

$$
\begin{gathered}
u(x, t)=\sum_{n=0}^{N-1} t^{-(m+n) / 2} \sum_{j=0}^{p+n+2} \ln ^{j} t\left[t^{(p+1) / 2} S_{n, j}\left(\frac{x}{2 \sqrt{t}}\right)+\Pi_{n, j}\left(\frac{x}{2 \sqrt{t}}\right) \exp \left(-\frac{|x|^{2}}{4 t}\right)\right] \\
+O\left(\left(|x|^{2}+t\right)^{-\rho N}\right)
\end{gathered}
$$

for each $N \geqslant p+1$ as $|x|^{2}+t \rightarrow+\infty$. In general, the exact formulas for $S_{n, j}(\eta)$ and $\Pi_{n, j}(\eta)$ are fairly cumbersome; however, by using the above proofs, one can derive them in particular cases. Note that, as shown by earlier investigations, asymptotic expansions in half-integer powers of $t$ are naturally intrinsic to solutions of the heat equation, see, for example, [19, Ch. X, §1] and [20].

In conclusion, following Poincaré's thesis "sans généralisation, la prévision est impossible" 4 (see his "La Science et l'Hypothèse", Ch. IX), it is appropriate to say that the immense variety of asymptotics of initial data together with the account of possible external sources of heat opens a wide field of further investigation of the long-time behavior of heat distribution by the above-presented method; in addition, other types of equations whose solutions have the form of convolutions can also be treated in a similar way.

\section{REFERENCES}

1. Danilin A.R. Asymptotic behaviour of bounded controls for a singular elliptic problem in a domain with a small cavity. Sb. Math., 1998. Vol. 189, No. 11. P. 1611-1642. DOI: $10.1070 /$ SM1998v189n11ABEH000364

\footnotetext{
4 "prevision is impossible without generalization"
} 
2. Denisov V. N. On the behavior of solutions of parabolic equations for large values of time. Russian Math. Surveys, 2005. Vol. 60, No. 4. P. 721-790. DOI: 10.1070/RM2005v060n04ABEH003675

3. Erdélyi A., Wyman M. The asymptotic evaluation of certain integrals. Arch. Rational Mech. Anal., 1963. Vol. 14, P. 217-260. DOI: 10.1007/BF00250704

4. Fourier J. Théorie Analytique de la Chaleur. Paris: Firmin Didot Père et Fils, 1822. 639 p. (in French)

5. Friedman A. Asymptotic Behavior of solutions of parabolic equations of any order. Acta Math., 1961. Vol. 106, No. 1-2. P. 1-43. DOI: 10.1007/BF02545812

6. Friedman A. Partial Differential Equations of Parabolic Type. New Jersey: Prentice-Hall, Englewood Cliffs, 1964. 347 p.

7. Gilkey P. B. Invariance Theory, the Heat Equation, and the Atiyah-Singer Index Theorem. Math. Lect. Ser., vol. 11. Delaware: Publish or Perish, Inc., Wilmington, 1984. 512 p.

8. Il'in A. M., Khas'minskii R.Z. Asymptotic behavior of solutions of parabolic equations and an ergodic property of non-homogeneous diffusion processes. Math. Sb. (N. S.), 1963. Vol. 60, No. 3. P. 366-392. (in Russian)

9. Il'in A. M. Matching of Asymptotic Expansions of Solutions of Boundary Value Problems. Transl. Math. Monogr., Vol. 102. Am. Math. Soc., 1992. 281 p.

10. Narasimhan T. N. Fourier's heat conduction equation: History, influence, and connections. Rev. Geophys., 1999. Vol. 37, No. 1. P. 151-172. DOI: 10.1029/1998RG900006

11. Kamin S., Peletier L. A., Vazquez J. L. A nonlinear diffusion-absorption equation with unbounded initial data. In: Nonlin. Diff. Eq. Equilib. Stat. Progr. Nonlinear Differential Equations Appl., vol. 7. Lloyd N.G., Ni W.M., Peletier L.A., Serrin J. (eds.) Boston, MA: Birkhäuser, 1992. Vol. 3, P. $243-263$. DOI: $10.1007 / 978-1-4612-0393-3 \_18$

12. Krzyżański M. Sur l'allure asymptotique des solutions d'équations du type parabolique. Bull. Acad. Polon. Sci., 1956. Vol. 4, No. 5. P. 247-251.

13. Lacey A. A., Tzanetis D. E. Global, unbounded solutions to a parabolic equation. J. Differential Equations, 1993. Vol. 101, No. 1. P. 80-102. DOI: 10.1006/jdeq.1993.1006

14. Li J. Heat equation in a model matrix geometry. C. R. Math. Acad. Sci. Paris, 2015. Vol. 353, No. 4. P. 351-355. DOI: 10.1016/j.crma.2014.10.024

15. Lieberman G. M. Second Order Parabolic Differential Equations. River Edge: World Scientific, 1996. 452 p. DOI: $10.1142 / 3302$

16. Poincaré H. Sur les intégrales irrégulières des équations linéaires. Acta Math., 1886. Vol. 8, No. 1. P. 295-344. DOI: 10.1007/BF02417092 (in French)

17. Reynolds A. Asymptotic behavior of solutions of nonlinear parabolic equations. J. Differential Equations, 1972. Vol. 12, No. 2. P. 256-261. DOI: 10.1016/0022-0396(72)90032-0

18. Tychonoff A. Théorèmes d'unicité pour l'équation de la chaleur. Math. Sb., 1935. Vol. 42, No. 2. P. 199216. (in French)

19. Widder D. V. The Heat Equation. New York: Academic Press, 1976. 267 p.

20. Zakharov S. V. Heat distribution in an infinite rod. Math. Notes, 2006. Vol. 80, No. 3. P. $366-371$. DOI: $10.1007 /$ s11006-006-0148-x

21. Zakharov S. V. Two-parameter asymptotics in the Cauchy problem for a quasi-linear parabolic equation. Asympt. Anal., 2009. Vol. 63, No. 1-2. P. 49-54. DOI: 10.3233/ASY-2008-0927 